\title{
Autonomous car and ride sharing: flexible road trains
}

\author{
(Vision Paper) \\ Niels Agatz \\ Rotterdam School of \\ Management \\ Erasmus University \\ The Netherlands \\ nagatz@rsm.nl \\ Dirk Christian Mattfeld \\ Business Information Systems \\ University of Braunschweig \\ Braunschweig, Germany \\ d.mattfeld@tu- \\ braunschweig.de \\ Ana L. C. Bazzan \\ Instituto de Informatica \\ UFRGS \\ Porto Alegre, RS, Brazil \\ bazzan@inf.ufrgs.br \\ Monika Sester \\ IKG \\ Leibniz University Hannover \\ Hannover, Germany \\ monika.sester@ikg.uni- \\ hannover.de \\ Ouri Wolfson \\ Computer Science \\ University of Illinois \\ Chicago, USA \\ wolfson@cs.uic.edu
}

\author{
Ronny Kutadinata \\ Infrastructure Engineering \\ University of Melbourne \\ VIC 3010, Australia \\ ronny.kutadinata@unimelb.edu.au
}

\author{
Stephan Winter \\ Infrastructure Engineering \\ University of Melbourne \\ VIC 3010, Australia \\ winter@unimelb.edu.au
}

\begin{abstract}
Since in many cities transport infrastructure is operating at or beyond capacity, novel approaches to organize urban mobility are gaining attraction. However, assessing the benefits of a measure that has disruptive capacity in a complex system requires a carefully designed research. This paper takes a recent idea for urban mobility - flexible road trains - and illustrates the computational and research challenges of realizing its full potential and describing its social, ecological and economical impact.
\end{abstract}

\section{CCS Concepts}

-Human-centered computing $\rightarrow$ Ubiquitous and mobile computing systems and tools; •Applied computing $\rightarrow$ Transportation;

\section{Keywords}

Urban mobility, vehicle platooning, autonomous vehicles

\section{CHALLENGES OF URBAN MOBILITY}

Urban growth as a global phenomenon, rising pressure on resources (e.g., space for/cost of transport infrastructure), and growing concerns about health (e.g., emissions, or ac-

Permission to make digital or hard copies of all or part of this work for personal or classroom use is granted without fee provided that copies are not made or distributed for profit or commercial advantage and that copies bear this notice and the full citation on the first page. Copyrights for components of this work owned by others than ACM must be honored. Abstracting with credit is permitted. To copy otherwise, or republish, to post on servers or to redistribute to lists, requires prior specific permission and/or a fee. Request permissions from permissions@ acm.org.

SIGSPATIAL'16, October 31-November 03, 2016, Burlingame, CA, USA

(C) 2016 ACM. ISBN 978-1-4503-4589-7/16/10 . \$15.00

DOI: http://dx.doi.org/10.1145/2996913.2996947 tive transport) require new ways to organize urban mobility as we know it. At the same time, progress in vehicle technology (e.g., electric mobility, autonomous driving, connected vehicles) and smart infrastructure (e.g., intelligent transportation systems, shared mobility) inspires such disruptive ideas. Visions of autonomously driving taxis or smarter shared forms of mobility are now pervasive, and they all rely on progress in information and communication technology: on sensing and data analytics for the autonomously driving vehicle, and on the connected vehicle for coordination and collaborative optimization.

But are these visions of novel mobility services really solving the social, ecological and economical challenges, or at least reducing their impact? Urban mobility is a complex system, and predicting the impact of a disruption in a complex system is scientifically and computationally a hard question. Therefore, in this paper we focus on one single recent idea: flexible road trains for urban public transport. ${ }^{1}$ Flexible road trains are a fleet of autonomously driving vehicles (pods) providing door-to-door service that have the option to form platoons. When in a platoon formation, the vehicles are physically connected by passages that allow passengers to perform en-route transfer between pods while on the move. In this paper, we apply a synoptic approach to explore their potential impact on urban transport, identifying the various challenges in providing objective and thorough evidence for this impact. Society may expect such evidencebased advice from academia, and yet this paper can only lay out a research agenda. Addressing big picture questions in an integrated manner is a hard problem.

\footnotetext{
${ }^{1}$ http://www.next-future-mobility.com/, https://youtu.be/IDgh29SqZzE
} 


\section{OPPORTUNITIES OF FLEXIBLE ROAD TRAINS}

Flexible road trains relate to a number of researched concepts, which are discussed in this section.

Autonomously driving vehicles may replace personally driven cars by mobility as a service [22]. They are equipped with sensors and mapping technology in order to autonomously plan routes and travel safely along these routes. Furthermore, once these autonously driving cars connect to each other and the infrastructure, coordination and collaboration between the vehicles becomes feasible. In particular, this allows the flexible road trains to perform high-precision onthe-fly pods-coupling processes during platoon formation.

Car sharing is an established mode of transport and means a collaborative use of a vehicle [6], thus reducing cost of ownership. In contrast, ride sharing (or carpooling), another established mode of transport, refers to the concurrent use of a vehicle, where typically the driver is the owner and the passengers are joining for some part of the ride [5, 13, 24]. Ride sharing has also been suggested between passengers and goods [10]. Combined, an autonomously driving vehicle can provide taxi services and thus is a shared car that even can be used in ridesharing mode (shared taxi, shuttle). If these vehicles are built ultra-light they are also called pods; some design studies even allow standing. ${ }^{2}$

Vehicle platoons are studied predominantly in highway environments $^{3}$ [3]. Their frequently cited benefits on highways are a decreased headway or increased capacity of the road, reduced air resistance or greater fuel economy, and safer travelling due to the autonomous driving mode at least of the followers. These benefits on highway traffic are not easily transferable to urban transportation and public transport. Although a flexible road train in urban environments does produce higher vehicle density within the platoon, the impact on traffic flow in urban road networks with their higher density of intersections remains unknown. Some research states a rise in throughput at intersections from urban platoons [12], but evidence beyond a single intersection is missing. Also, compact cars have been shown to positively impact on urban road capacity [20], hence, compact lightweight pods for public transport may have a similar impact. However, urban platoons have not been reported to substantially help reducing air resistance or fuel consumption; quite to the contrary they will require compromises on route choices. Urban platoons have been suggested for a different purpose though, namely for collecting and redistributing autonomously driving pods that otherwise may operate only in defined environments [17].

Flexible road trains combine the benefits of these three concepts. They provide a door-to-door service such as taxi/ ride-sharing; they are able to form platoons to allow the exchange of passengers, goods, or resources (batteries) on the fly; and they are autonomously driving to enable precise coupling, coordination, and collaboration. However, since also a higher degree of organization is required to facilitate platooning between pods, this perceived improvement may be balanced by higher waiting times and detour costs compared to pure car sharing mode (autonomous taxi). Detours are

\footnotetext{
${ }^{2}$ http://www.next-future-mobility.com/

${ }^{3}$ Mostly trucks: http://www.sartre-project.eu, https:// eutruckplatooning.com, http://path.berkeley.edu/research/ automated-and-connected-vehicles/truck-platooning
}

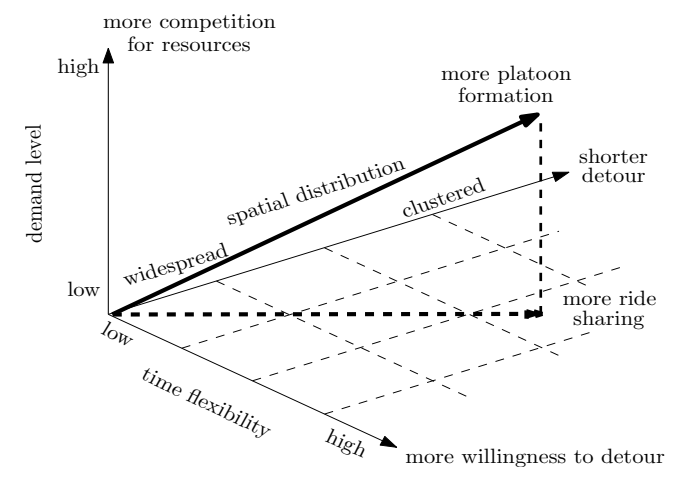

Figure 1: Road train demand scenarios.

also indicating a rise of vehicle-kilometers, and thus higher fuel costs and emissions. So, overall, such an idea should be investigated before implementation. Figure 1 shows the theoretical demand scenarios that flexible road trains can serve. From one end, a widespread and low demand scenario with low time flexibility (almost no detour allowance) potentially reduces the operation of the flexible road trains to a pure car sharing service. On the other extreme, where there is a high and clustered demand scenario with high time flexibility, a complex platooning coordination is required to efficiently manage the fleet.

\section{REALIZATION CHALLENGES}

In this section we present a list of areas in which computational methods can contribute to flexible road trains.

\subsection{Routing, detours and platoons}

Maximizing the advantages offered by the flexible road trains arguably requires a high degree of organization. In particular, a properly organized flexible road train service may offer an attractive public transport alternative due to its perceived convenience by the public. However, this still has to be balanced by the minimization of operation cost. Therefore, this leads to a challenging optimization problem where the following trade-offs are faced: (a) minimizing the number of vehicles on the road to reduce congestion might lead to a service where most of the customers suffer great delay/waiting/detour, which reduces the attractiveness of the service; (b) maximizing convenience leads to the extreme case - a taxi-like service (i.e. non-shared direct trips), which in turn does not help alleviating congestion and reducing the total travel time; (c) minimizing the total travel time (of all passengers) might require an optimal trade-off of the two points above, but passenger convenience is not guaranteed (e.g. numerous transfers might be required); and (d) minimizing operating cost might lead to a large number of unserved customers (e.g. due to lack of pods). These challenges are compounded by the lack of mathematical formulation of platooning and en-route transfer. The formulation has to consider the spatio-temporal constraint of platoon formation, the minimum amount of time required for transfer, and transfer restrictions (e.g. expecting disadvantaged passengers to transfer is unreasonable).

Hence, the challenges here are: formulating an optimization problem that allows platooning and transfer; revisiting the concept of "shortest" path by considering a more com- 
plex objective function that quantifies the relative importance of various considerations; and obtaining an acceptable trade-off such that the identified potential benefits of flexible road trains are achieved. The first challenge can be seen as an extension of the dial-a-ride problem with transfers [15]. To address the second and third challenges, a potential approach is to set one aspect as a constraint/target to be reached while optimizing the other aspects. For instance, it is possible to minimize the operating costs such that a certain service level, in terms of waiting and travel time of each individual passenger, is reached.

\subsection{Real-time planning issues}

As a flexible transport service, the system has to be able to serve requests on-demand. Choosing an appropriate scheme of accepting such ad-hoc demands also require a thorough investigation. For instance, the booking has to be made some period of time before the trip, ranging from 10 minutes up to 180 minutes prior [14]. The impact of such constraint can be significant from the organization perspective. If the organization is inadequate, these ad-hoc requests might significantly reduce the routing optimality. Therefore, it is important to devise a suitable algorithm to handle these requests.

There are many algorithms in the literature to handle ad-hoc requests $[18,8,2,21,19]$, ranging from simple to complex ones. However, simple heuristic methods, such as greedy insertion, are likely to result in suboptimal solutions. In more complex ones, typically some stochastic information about the demand pattern is required, which might not be available in such a novel transport mode. In addition, servicing a whole city with potentially thousands of demands and pods might lead to a scalability issue, which encourages some degree of decentralized planning. Hence, a novel framework/algorithm might be required to accommodate requests on-demand in flexible road trains.

\subsection{Strategic supply design}

In order to assist in handling ad-hoc requests, a suitable pods allocation strategy is required to improve the coverage of the whole service area. From a set of potentially possible parking lots, a coverage model selects a subset of minimum cardinality satisfying some externally specified maximum supply time. The coverage is done in a way such that all possible customer locations are within a reach from at least one of the subset of selected parking lots.

The service network design determines the number of pods to be placed at parking lots at the hours of a day. The expected demand of pods within the reach of a parking lot at a certain hour of the day is either determined from historic trip data or is approximated from a respective survey (see Section 3.5). This temporal data reports the pickup and return of pods in the granularity of the reach of parking lots in terms of time dependent origin/destination matrices. The autonomy of the pods allows the respective service network design model determines the recirculation of pods (as in [1]) returned from customers to parking lots such that some future expected demand is met. Additionally, the platooning capability of the pods can potentially be utilized to minimize the energy consumption of the pods redistribution.

\subsection{Sensing and information}

Functioning of the system heavily relies on different kinds of data. As fundamental information, spatial data about the road network has to be available. For the autonomous cars to navigate precisely, especially when it comes to the docking maneuvers, precise localization in the low dm-range is needed [4]. Today, the automotive industry is working on data formats to store this kind of maps [11], as well as on methods to acquire the information (e.g., road lanes, lane markings, landmarks).

Secondly, real time information about the travelers is required: this relates on the one hand to the actual travel demand (start and destination, time window, additional constraints) and also information about personal preferences, which can be used as matching constraints for the grouping of passengers.

Finally, information about the traffic situation has to be available, especially for routing purposes. To this end, either data from traffic infrastructure (inductive loops, CCTV cameras) can be used, but also information from social media and crowdsourcing. This leads to dynamic information about demand patterns in space and time in a city. Challenges lie in the processing of all this information, which is constantly streaming, especially related to representation of data in dynamic maps, learning patterns from raw data, parallel/distributed/decentralized processing, application of Hadoop/Spark ecosystems, as well as protection of individual privacy.

\subsection{Human factors}

Acceptance of novel mobility services will depend also on human factors in the system design, i.e., how the systemimmanent information is conveyed to and consumed by the travelers. Human factors cannot be underestimated $[7,16]$, and flexible road trains bring up two central aspects of human factors: one is the design of a safe and convenient onthe-fly transfer between pods in the train before it splits up again, and the other is the usability of the trip information provided in advance and during the travel.

\subsection{Economic feasibility and pricing}

Both optimization as well as human factors can further be complicated by the introduction of pricing. Although operation cost minimization has been considered, this does not consider the income of the service. Allowing a higher cost that leads to a higher attractiveness can be justified by an even higher income being generated. In addition, dynamic pricing to reflect the user's preferences (relating to transfer, trip-sharing, and flexibility) might further increase the attractiveness. However, this might add more complications to an already complex routing and platooning problem. Thus, a carefully designed pricing scheme might be one of the most deciding factors in the feasibility of the flexible road trains. Human factors, on the other hand, require a transparent and simple pricing schema.

\subsection{Simulation for evaluation}

As a complex system, the benefits of flexible road trains on urban transportation is not directly predictable. Typically, detailed examinations of new systems in transportation is carried out by using simulation prior implementation $[9,19,23,20]$. Due to the heavy infrastructure commitment required to realize this concept, simulation becomes an attractive approach to investigate the potential benefits of the flexible road trains.

A simulation experiment is a powerful tool to investigate 
various performances of the flexible road trains and, simultaneously, its impacts on traffic. For instance, it is important to examine the resulting routes proposed by the optimization algorithm: the number of platoons formed, their length, and the duration of the platoon formation. As another example, a comparison study can be carried out to analyze whether increased traffic flows are detected in the presence of flexible road trains. As such, a micro simulator is necessary, which might still need to be properly developed and validated.

\section{FINAL REMARKS AND OUTLOOK}

People's mobility is a derived demand, required for access and equity. Since in many cities transport infrastructure is operating at or beyond capacity, disruptive modes of mobility are suggested. The paper addresses the challenges of introducing such a technology using the example of flexible road trains. Computational, economic, ecological as well as social aspects are considered. It contributes to a research agenda in smart cities from a computational perspective.

\section{Acknowledgments}

This paper is an outcome of Dagstuhl Seminar 16091: Computational challenges in cooperative intelligent urban transport. Supports from ARC LP120200130, NSF grants IIS1213013 and IIP-1534138 are acknowledged.

\section{REFERENCES}

[1] A. L. C. Bazzan, M. d. B. do. Amarante, and F. B. da Costa. Management of demand and routing in autonomous personal transportation. Journal of Intelligent Transportation Systems, 16(1):1-11, 2012.

[2] R. Bent and P. Van Hentenryck. Scenario-based planning for partially dynamic vehicle routing with stochastic customers. Operations Research, 52:977-987, 2004.

[3] C. Bergenhem, H. Pettersson, E. Coelingh, C. Englund, S. Shladover, and S. Tsugawa. Overview of platooning systems. In A. Stevens and E. Chung, editors, 19th ITS World Congress.

[4] C. Brenner. Vehicle localization using landmarks obtained by a lidar mobile mapping system. Int. Arch. Photogramm. Remote Sens, 38:139-144, 2010.

[5] N. D. Chan and S. A. Shaheen. Ridesharing in north america: Past, present, and future. Transport Reviews, 32(1):93-112, 2012.

[6] B. Cohen and J. Kietzmann. Ride on! mobility business models for the sharing economy. Organization Ef Environment, 27(3):279-296, 2014.

[7] E. Fishman, S. Washington, N. Haworth, and A. Watson. Factors influencing bike share membership: An analysis of melbourne and brisbane. Transportation Research Part A: Policy and Practice, 71:17-30, 2015.

[8] M. Gendreau, F. Guertin, J.-Y. Potvin, and E. Taillard. Parallel tabu search for real-time vehicle routing and dispatching. Transportation Science, 33:381-390, 1999.

[9] M. Horn. Fleet shceduling and dispatching for demand-responsive passenger services. Transportation Research Part C, 10:35-63, 2002.
[10] R. Kutadinata, R. G. Thompson, and S. Winter. Cost-efficient co-modal ride-sharing scheme through anticipatory dynamic optimisation. In 23rd World Congress on Intelligent Transportation Systems. ITS Australia.

[11] J. Levinson, M. Montemerlo, and S. Thrun. Map-Based Precision Vehicle Localization in Urban Environments, pages 121-128. MIT Press, 2008.

[12] J. Lioris, R. Pedarsani, F. Y. Tascikaraoglu, and P. Varaiya. Platoons of connected vehicles can double throughput in urban roads. Report, arxiv.org, 2015.

[13] S. Ma, Y. Zheng, and O. Wolfson. Real-time city-scale taxi ridesharing. IEEE Transactions on Knowledge and Data Engineering, 27(7):1782-1795, 2015.

[14] J. Mageean and J. D. Nelson. The evaluation of demand responsive transport services in Europe. Journal of Transport Geography, 11:255-270, 2003.

[15] R. Masson, F. Lehuédé, and O. Péton. The dial-a-ride problem with transfers. Computers $\&$ Operations Research, 41:12-23, 2014.

[16] M. McGregor, B. Brown, and M. GlÃüss. Disrupting the cab: Uber, ridesharing and the taxi industry. Journal of Peer Production, (6 - Disruption and the Law), 2015.

[17] M. Parent and P. Daviet. Automatic driving for small public urban vehicles. In I. Masaki, editor, Intelligent Vehicles '93 Symposium, pages 402-407. IEEE, 1993.

[18] H. N. Psaraftis. A dynamic-programming solution to the single vehicle many-to-many immediate request dial-a-ride problem. Transportation Science, 14(2):130-154, 1980.

[19] N. Ronald, R. Thompson, J. Haasz, and S. Winter. Determining the viability of a demand-responsive transport system under varying demand scenarios. In 6th ACM SIGSPATIAL International Workshop on Computational Transportation Science, 2013.

[20] N. Ronald, A. Vishwanath, and S. Winter. Simulating city-wide impacts of microcars: preliminary results. In L. Padgham and M.-R. Namazi-Rad, editors, First Workshop on Agent Based Modelling of Urban Systems (ABMUS 2016).

[21] N. Seconmandi and F. Margot. Reoptimization approaches for the vehicle-routing problem with stochastic demands. Operations Research, 57(1):214-230, 2009.

[22] A. Spickermann, V. Grienitz, and H. A. von der Gracht. Heading towards a multimodal city of the future?: Multi-stakeholder scenarios for urban mobility. Technological Forecasting and Social Change, 89:201-221, 2014.

[23] A. Vishwanath, H. S. Gan, S. Kalyanaraman, S. Winter, and I. Mareels. Personalised public transportation: a new mobility model for urban and suburban transportation. In Proceedings of the 17th International IEEE Conference on Intelligent Transportation Systems, pages 1831-1836, 2014.

[24] S. Winter and S. Nittel. Ad-hoc shared-ride trip planning by mobile geosensor networks. International Journal of Geographical Information Science, 20(8):899-916, 2006. 